\title{
The temperature dependence of the inelastic scattering time in InGaN grown by MOVPE
}

\author{
A. Yildiz ${ }^{1}$ and M. Kasap ${ }^{2}$ \\ ${ }^{1}$ Department of Physics, Faculty of Science and Arts, Ahi Evran University, Kirsehir 40040, Turkey \\ E-mail: yildizab@gmail.com \\ ${ }^{2}$ Department of Physics, Faculty of Science and Arts, Gazi University, Teknikokular, Ankara 06500, Turkey
}

Received September 18, 2009, revised December 24, 2009

\begin{abstract}
Low temperature electrical measurements of the resistivity, the Hall effect and the magnetoconductivity were performed on an InGaN sample having an electron concentration far above the critical value for the metalinsulator transition. Weak localization effect and two-band model were used to analyze the magnetoconductivity data. The temperature dependence of the inelastic scattering time was extracted from the magnetoconductivity data at low temperatures. It was found that the inelastic scattering time is proportional to $T^{-1.63}$, suggesting that electron-electron interactions are dominant.
\end{abstract}

PASC: $72.20 . \mathrm{My}$ Galvanomagnetic and other magnetotransport effects;

72.20.Fr Low-field transport and mobility; piezoresistance;

72.80.Ey III-V and II-VI semiconductors.

Keywords: weak localization; electron-electron interaction; two-band model; inelastic scattering time; positive magnetoconductivity; impurity band; InGaN; MOVPE.

\section{Introduction}

Wide band gap group III-nitride semiconductors have attracted wide attention in view of their application in electronic devices such as short-wavelength light-emitting diodes (LEDs) [1], modulation-doped field effect transistors (MODFETs) [2], and ultra-violet (UV) detectors [3].

The study of magnetic field related electron transport in group III-nitride semiconductors is needed for their electronic device applications. The temperature dependence of inelastic scattering time $\left(\tau_{i}\right)$ is considered to be an important parameter in the physics of the electronic devices. In order to investigate device performance, the behavior of the $\tau_{i}$ needs to be investigated. Improving device performances requires an understanding of how $\tau_{i}$ depends on temperature. For a material, the $\tau_{i}$ can be found from the magnetoconductivity (MC) data. To the best of the authors' knowledge there is no report available in the literature on the inelastic scattering time of InGaN.

In a previous work [4], we showed that $\mathrm{InGaN}$ layers have a high bowing parameter $\sim 3.6 \mathrm{eV}$, which indicates presence of disorder and defects in the structure of InGaN. These defects can induce localized levels in an impurity band. If carrier concentration of a material is above the metal-insulator transition (MIT) concentration, the conduction of the material is mainly described by metallic con- duction in a degenerate band. The MIT is generally explained by the increase in carrier concentration and formation of a degenerate band due to doping [5]. However, the intrinsic as well as extrinsic donors should cause to a high carrier concentration. The observed metallic conduction in unintentionally doped $\mathrm{InGaN}$ system may be explained by a large number of nitrogen vacancies which act as donors and lead to degeneracy [6].

In normal metals, the Fermi wavelength is much smaller than the mean free path of the carriers, and the Boltzmann approach can successfully describe the transport properties [7-9]. However, structural or composition disorders are increased, the mean free path becomes small and eventually it may become smaller than Fermi wavelength. In high degenerate semiconductors, the mean free path between collisions becomes small and quantum effects become important, as structural or compositional disorder increases in the material. In this case, the Boltzmann approach may not successfully describe the transport properties of the material. The investigation of the low temperature electrical conductivity of different disordered electronic systems indicated that various corrections terms needs to be added to the classical Boltzmann conductivity [7-9]. These corrections can be distinguished through their different dependences on temperature and magnetic field. 
In this paper, we investigated the low temperature electrical properties of an InGaN layer grown by MOVPE. The aim of the work is to the determination of the temperature dependence of $\tau_{i}$ from MC data of the investigated sample.

\section{Experimental}

The $\mathrm{In}_{0.12} \mathrm{Ga}_{0.88} \mathrm{~N}$ epilayer presented in this work was grown in an atmospheric pressure cold-wall vertical MOVPE reactor with a shower configuration. The standard heterostructure included a $80-100 \mathrm{~nm}$ thick GaN buffer grown at $510{ }^{\circ} \mathrm{C}$, a $600 \mathrm{~nm}$ thick $\mathrm{GaN}$ layer deposited at $1080{ }^{\circ} \mathrm{C}$ (typical V/III ratio was about 7000), and an $\mathrm{In}_{0.12} \mathrm{Ga}_{0.88} \mathrm{~N}$ alloy deposited at $800{ }^{\circ} \mathrm{C}$ with different conditions, in order to vary In content. The thickness of $\mathrm{In}_{0.12} \mathrm{Ga}_{0.88} \mathrm{~N}$ epilayer is $200 \mathrm{~nm}$. Details of the growth procedures were given elsewhere [10]. The In composition was studied by $x$-ray diffraction (standard $\theta / 2 \theta$ diffractometer) assuming that the lattice parameter varies linearly with the In fraction according to Vegard's law [10].

For resistivity and Hall effect measurements by the van der Pauw method, square shaped $(5 \times 5 \mathrm{~mm})$ sample was prepared with four contacts in the corners. Using annealed indium dots, ohmic contacts to the sample were prepared and their ohmic behavior was confirmed by the currentvoltage characteristics. Measurements were made at temperature steps over temperature range 20-350 K using a Lake Shore Hall effect measurement system (HMS). At each temperature step, the Hall coefficient (with maximum $5 \%$ error) and resistivity (with maximum $0.2 \%$ error in the studied range) were measured for both current directions, both magnetic field directions that were perpendicular to the surface and all the possible contact configurations at 14 magnetic field steps between 0 and $1.4 \mathrm{~T}$ (with $0.1 \%$ uniformity).

\section{Results and discussion}

Figure 1 shows the temperature dependence of the electrical resistivity. At low temperatures, the sample shows negative temperature coefficient $(d \rho / d T<0)$ of the resistivity, while the resistivity tends to saturate $(d \rho / d T \rightarrow 0)$ at high temperatures. Normally, one can expect that electron-phonon scattering becomes important at high temperatures. However, in our case, Hall mobility and carrier concentration are almost temperature independent. We may consider that residue impurity and dislocation scattering are temperature independent for a degenerate electron system, causing the resistivity to saturate [11].

We characterize the transport properties of the sample in terms of the resistivity ratio, $\rho_{r}=\rho(20 \mathrm{~K}) / \rho(300 \mathrm{~K})$. The temperature dependence $\rho(T)$ of the investigated sample is very weak with $\rho_{r}=1.13$. One can expect that the value of $\rho_{r}$ becomes very low on metallic side of the MIT. The critical density $\left(n_{c}\right)$ for the MIT is obtained by using the re-

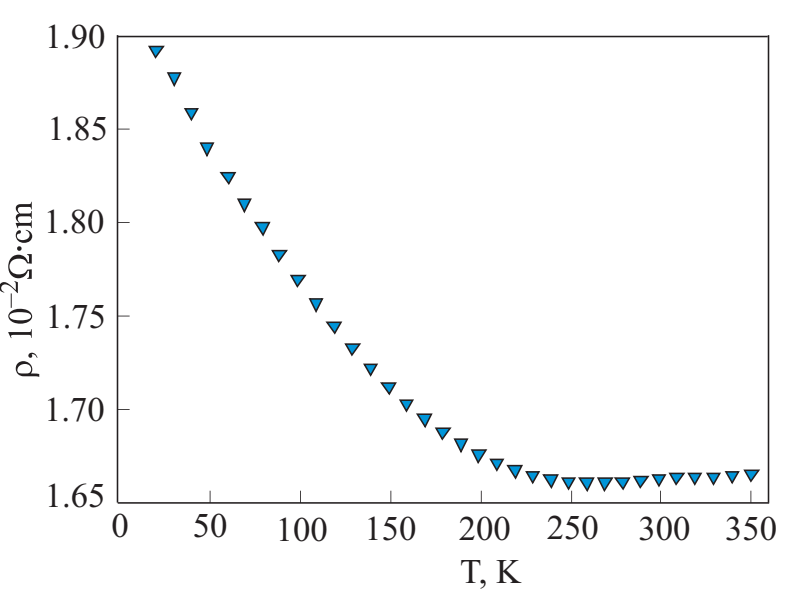

Fig. 1. Temperature dependence of the resistivity of InGaN in a temperature range $20-350 \mathrm{~K}$.

lation $n_{C}=\left(0.25 / a_{B}^{*}\right)^{3}$, where $a_{B}^{*}$ is Bohr radius. The $a_{B}^{*}$ is given with relation $a_{B}^{*}=4 \pi \varepsilon_{0} \varepsilon \hbar^{2} / m^{*} e^{2}$, where $\varepsilon_{0}$ is the permittivity of vacuum, $\varepsilon$ is the static dielectric constant, $\hbar$ is Planck's constant and $e$ is the electron charge. By using an iterational method [12], the values of $a_{B}^{*}$ of $\operatorname{In}_{x} \mathrm{Ga}_{1-x} \mathrm{~N}$ alloys as a function of $x$ can be calculated with the values of effective masses $m^{*}=0.22 \mathrm{~m}_{0}$ and $0.115 \mathrm{~m}_{0}$, and the static dielectric constants of $\varepsilon=10.4$ and 15.3 for $\mathrm{GaN}$ and $\mathrm{InN}$, respectively. We obtained $n_{C}$ as $7.1 \cdot 10^{17} \mathrm{~cm}^{-3}$ for $x=0.12$. The investigated sample has a carrier concentration $(n)$ of $1.1 \cdot 10^{19} \mathrm{~cm}^{-3}$, which is far above the $n_{c}$ of $7.1 \cdot 10^{17} \mathrm{~cm}^{-3}$ for the MIT. We conclude, therefore, that the investigated sample is on the metallic side of the MIT. In our case, the observed features suggest that the localization-interaction model for disordered metallic systems near the MIT can be used for an explanation of low-temperature metallic transport in the sample $[6,9,13]$.

In order to achieve deeper understanding the low temperature carrier transport properties of the sample, we measured the magnetic field dependence of resistivity of the sample at three selected temperatures. Figure 2 shows the variation of the $\Delta \sigma(B)$ with magnetic field at 20,40 and $50 \mathrm{~K}$ for the sample in which the points represent the experimental data and the solid lines display the theoretically best fitted values obtained by using Eq. (1). In Eq. (1), the $\Delta \sigma(B)$ is described as $\Delta \sigma(B)=[\sigma(B)-\sigma(0)] / \sigma^{2}(0)$. It is seen that the $\mathrm{MC}$ is relatively weak. In a previous work [6], we observed a similar MC behavior in InGaN. As observed in Fig. 2, the $\mathrm{MC}$ is positive, but the magnitude decreases systematically with increasing temperature, and one may expect that it becomes negligible above $\sim 50 \mathrm{~K}$. One can assign this remarkable temperature dependence to a positive $\mathrm{MC}$ arising from destruction of weak localization (WL) by the application of magnetic field [9]. Kawabata [14] discussed the correction to the conductivity in the WL regime.

The MC in non-magnetic degenerate semiconductors can be originated from several effects, including weak 


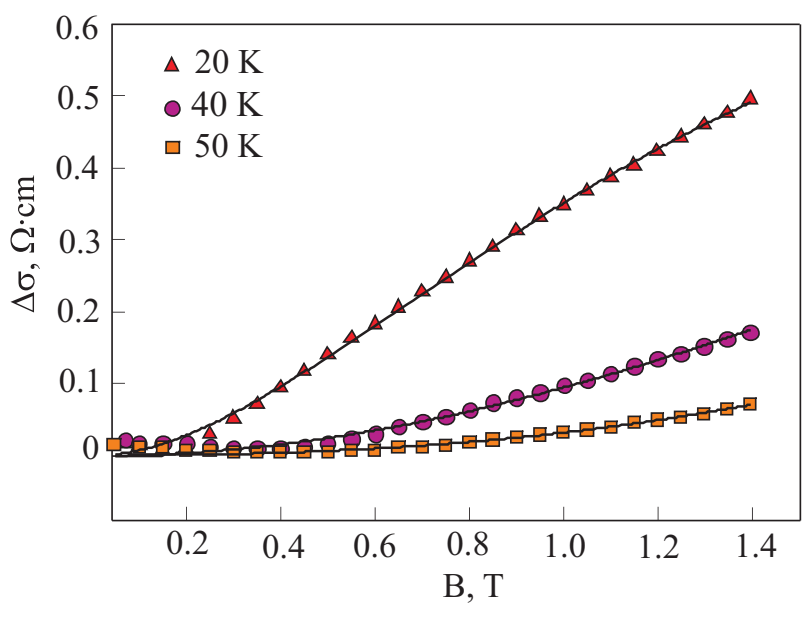

Fig. 2. Magnetoconductivity at three temperatures for InGaN. The solid lines represent the least-squares fits to the Eq. (1), the symbols are the experimental results.

antilocalization (WAL) effect, Lorentz force, electronelectron interactions (EEI) and WL effects [7-9]. The corrections of the first three effects to the $\mathrm{MC}$ are negative. On the other hand, WL effects result in positive MC.

The presence of spin-orbit scattering top of the valence band in the material, the WAL effect becomes important. However, spin-orbit scattering depends on the atomic number of doping atom. If the impurity atoms are heavy atoms, the spin-orbit scattering will contribute significantly to the MC [7]. Otherwise, the contribution to the MC due to the WAL effect can be ignored. A magnitude for the spin-orbit scattering time [7] can be estimated from the expression $\tau_{s 0} \sim \tau_{0}(137 / Z)$ where $Z$ is the atomic number and where $\tau_{0}$ is the elastic scattering time. For the case of strong spin-orbit scattering, $\tau_{s 0}$ is low and the WAL contribution produces a decrease in the zero magnetic field conductivity with increasing temperature. The MC resulting from Lorentz force should not be rule out due to the presence of impurity band in the disordered semiconductor materials. A two-band model describes this effect [8]. The EEI effect considers the modification of the effective Coulomb interaction in the presence of disorders in the systems [9]. It changes the density of states. As a result, the zero magnetic field conductivity of the system increases with increasing temperature.

Since there is quantum interference between the electronic waves at low temperatures, the back-scattering probability of electrons will be enhanced. This leads to a result in the terms of weak localization of electrons. When an external magnetic field is applied, the WL is suppressed. Because the magnetic field suppresses the wave coherence, thus, quantum interference effects are reduced and this results in positive MC [9]. Weak localization contribution causes an increase in the conductivity with increasing temperature.
In our situation, since the $\mathrm{MC}$ is positive, we can rule out the contributions to MC due to the WAL effect and EEI in undoped InGaN sample. Since the formation an impurity band in InGaN can be expected due to defects in its structure [4], we can consider the contribution of the Lorentz force to the MC. Then the MC at a given temperature, including both the WL effect and two-band model can be given by the expression $[8,14]$,

$$
\Delta \sigma(B)=\frac{e^{2}}{2 \pi^{2} \hbar} \sqrt{\frac{e B}{\hbar}} f_{3}\left(\frac{B}{B_{i}}\right)-\frac{a^{2} B^{2}}{1+b^{2} B^{2}},
$$

where $B_{i}=\hbar / 4 e D \tau_{i}$ refers to the inelastic scattering fields, $D$ is the diffusion coefficient, and the temperature dependence of inelastic scattering time has the relationship $\tau_{i} \sim T^{-p}$ (where $p$ is an index depending on scattering mechanism nature). Here $f_{3}(\delta)$ is expressed by a polynomial proposed by Baxter et al. [15],

$$
\begin{aligned}
f_{3}(\delta)= & 2\left(\sqrt{2+\frac{1}{\delta}}-\sqrt{\frac{1}{\delta}}\right)+\frac{1}{48}\left(2.03+\frac{1}{\delta}\right)^{-3 / 2}- \\
& -\left[\left(\frac{1}{2}+\frac{1}{\delta}\right)^{-1 / 2}+\left(\frac{3}{2}+\frac{1}{\delta}\right)^{-1 / 2}\right] .
\end{aligned}
$$

The parameters $a$ and $b$ are functions of conductivity and concentration of each group of carriers [8],

$$
\begin{gathered}
a=\frac{1}{\sqrt{\rho_{0}}} \frac{1}{e}\left(\frac{\sigma_{01}}{n_{1}}+\frac{\sigma_{02}}{n_{2}}\right) \frac{\sqrt{\sigma_{01} \sigma_{02}}}{\sigma_{01}+\sigma_{02}}, \\
b=\frac{1}{e}\left(\frac{n_{1}-n_{2}}{n_{1} n_{2}}\right) \frac{\sigma_{01} \sigma_{02}}{\sigma_{01}+\sigma_{02}},
\end{gathered}
$$

where $n_{1}$ and $n_{2}$ are the carrier concentrations in different bands, and $\sigma_{01}$ and $\sigma_{02}$ are conductivities for each group of carriers.

The validity of Eq. (1) is restricted to low magnetic fields under three conditions [14]

$$
\begin{gathered}
\hbar / m^{*} v_{F}^{2} \tau_{0}<1, \\
e B \tau_{0} / m^{*}<1,
\end{gathered}
$$

and

$$
\sqrt{e B / \hbar} l_{0}<1,
$$

where $v_{F}$ is the Fermi velocity, $l_{0}$ is the elastic mean free path, and $\tau_{0}=l_{0} / v_{F}$ the elastic scattering time.

The fitting of the MC data was performed by considering the contributions to $\mathrm{MC}$ due to WL effect and two-band model with Eq. (1) at low temperatures. Here, $B_{i}$, $a$ and $b$ are considered to be fitting parameters. They are collected in Table 1. The values of $a$ and $b$ decrease with increasing temperature. The fitted results of Eq. (1) to the MC data are very well as shown solid line in Fig. 2. This 
confirms that the positive component of the $\mathrm{MC}$ in the sample originates from a destructive effect of the magnetic field on the wave coherence. Then the inelastic scattering length, defined as $L_{i}=\sqrt{D \tau_{i}}=\sqrt{\hbar / 4 e B_{i}}$, can be determined for all temperatures. The values of the $L_{i}$ are also given in Table 1.

Table 1

\begin{tabular}{c|c|c|c|c|c}
\hline \hline$T, \mathrm{~K}$ & $a$ & $b$ & $B_{i}, \mathrm{~T}$ & $L_{i}, \mathrm{~nm}$ & $\tau_{i}, \mathrm{ps}$ \\
\hline 20 & 0.3817 & 0.5216 & 0.2283 & 27.207 & 4.991 \\
\hline 40 & 0.2949 & 0.5088 & 0.6414 & 16.232 & 1.612 \\
\hline 50 & 0.2845 & 0.5036 & 0.9625 & 13.251 & 1.121 \\
\hline \hline
\end{tabular}

Figure 3 shows the $B_{i}$ variation with temperature at all temperatures. We obtain that temperature dependence of $B_{i}$ is $B_{i}=1.602 \cdot 10^{-3} \cdot T^{1.63}$, i.e., the inelastic scattering time $\tau_{i} \sim T^{-1.63}$ at temperatures between 20 and $50 \mathrm{~K}$. As mentioned previously, $\tau_{i}$ is given by $\tau_{i} \sim T^{-p}$. For electron-phonon scattering $p$ is expected to be equal to 3 , while in the case of inelastic electron-electron scattering, $p=2$ and 1.5 for the clean and dirty limit, respectively [16]. Our result is in agreement with the theoretical results introduced for electron-electron scattering. Therefore, electronelectron scattering can be considered to be dominant in the sample. Since the obtained for value of $p$ in the vicinity of 1.5 , we assume that a $p=1.5$ dependence would be better than a $p=2$. We may expect that the inelastic electronelectron scattering is in dirty limit for the sample.

We should check the criteria (Eq. (5)-Eq. (7)) for the validity of Eq. (1) in the investigated sample. For applicability of Eq. (1) in the investigated sample, these criteria are to be satisfied. The $\tau_{0}$ can be obtained from the conductivity at $T=0 . \sigma_{0}(T=0)$ generally equals to the Boltzmann conductivity,

$$
\sigma_{B}=n e^{2} \tau_{0} / m^{*}
$$

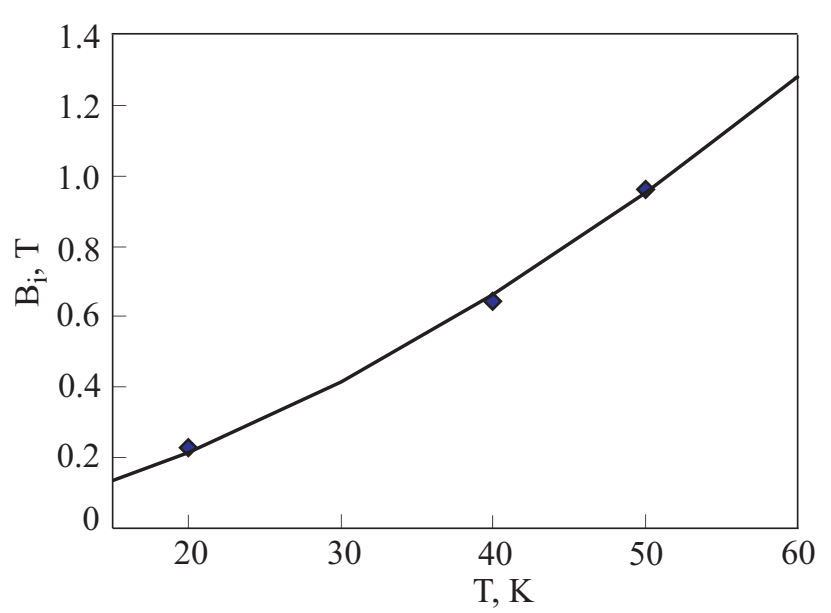

Fig. 3. The fitted values of inelastic scattering field $B_{i}$ as a function of temperature for $\mathrm{InGaN}$. The solid line is drawn proportional to $T^{1.63}$.
However, corrections to the zero temperature conductivity $\left(\sigma_{0}\right)$ have to be considered in the present sample, which is given by [17]

$$
\sigma_{0}=\sigma_{B}\left[1-\frac{C}{\left(k_{F} l_{0}\right)^{2}}\right]
$$

where $k_{F}$ is the Fermi wave vector and $C$ is a numerical coefficient ranging between 1 and 3 . In our calculations, $C$ was taken equal to 3 , as suggested by Mott [18]. The solution of Eq. (9), taking into account $\sigma_{0}=49.6 \Omega^{-1} \cdot \mathrm{cm}^{-1}$, gives $l_{0}=3.16 \mathrm{~nm}$ and $k_{F} l_{0}=2.28$. The first criterion $\left(k_{F} l_{0}\right)^{-1}=\hbar / m^{*} v_{F}^{2} \tau_{0}<1$ is fulfilled, i.e., $k_{F} l_{0}>1$. Since the investigated sample has a low Hall mobility of $35 \mathrm{~cm}^{2} /(\mathrm{V} \cdot \mathrm{s})$, the condition of $\mu B=e B \tau_{0} / m^{*}<1$ is satisfied in the range of the analyzed fields $(B=0-1.4 \mathrm{~T})$. It can be seen that Eq. (7) is satisfied over the entire range for $l_{0}=$ $=3.16 \mathrm{~nm}$. Therefore the sample is expected to have the MC data expressed by Eq. (1).

Here we recall that the thickness of the sample is $t=$ $=200 \mathrm{~nm}$, which is far above the estimated inelastic scattering length $L_{i}$, given in Table 1 , and then the assumption of a three-dimensional (3D) limit is consistent [9]. In the metallic side of the metal-insulator transition, the conductivity of a degenerate semiconductor increases with decreasing temperature as is typical for a good metal. However, due to structural or compositional disorder in the material, the conductivity of the material decreases with decreasing temperature at low temperatures. It is widely accepted that the electron transport properties of such materials can be explained with WL and EEI phenomenon at low temperatures [9]. The EEI contribution seems always to dominate in 3D disordered systems, resulting in an increase of the zero magnetic field resistivity with decreasing temperature. In the absence of a magnetic field, the corrections to Boltzmann conductivity due to WL and EEI for 3D disordered metallic systems can be given $[9,19,20]$,

$$
\begin{gathered}
\Delta \sigma(T)=\frac{e^{2}}{2 \pi^{2} \hbar} \frac{\eta}{\tau_{i}^{1 / 2}}+ \\
+\frac{e^{2}}{\hbar}\left(\frac{1.3}{4 \pi^{2}}\right)\left(\frac{k_{B} T}{2 \hbar D}\right)^{1 / 2}\left[\frac{4}{3}-\left(\frac{3}{2}\right) \gamma F_{\sigma}\right]
\end{gathered}
$$

where $\eta$ is a constant and $k_{B}$ is Boltzmann's constant. The diffusion coefficient, $D$, can be estimated from the relation $\left(\sigma_{0}=2 e^{2} D N(0)\right)$, where $N(0)$ is the concentration of states $\left(N(0)=m^{*} k_{F} / 2 \pi^{2} \hbar^{2}\right)$ [13]. The value of $D$ was obtained as $1.6 \mathrm{~cm}^{2} / \mathrm{s} . \gamma F_{\sigma}$ is the Coulomb interaction parameter where $\gamma$ is taken as 1.95 for InGaN [13]. The quantity $F_{\sigma}$ is related to the Fermi-liquid parameter $F$ by [20]

$$
F_{\sigma}=\left(-\frac{32}{3}\right)\left[1-\frac{3 F}{4}-\left(1-\frac{F}{2}\right)^{3 / 2}\right] F^{-1},
$$


The Fermi-liquid parameter $F$ is expressed as $[9,20]$,

$$
\begin{gathered}
F=\frac{1}{y} \ln (1+y), \\
y=\left(\frac{2 k_{F}}{K}\right)^{2},
\end{gathered}
$$

where $K$ is the screening wave factor which is given [9],

$$
K=\left(\frac{12 \pi n m^{*} e^{2}}{\varepsilon \hbar^{2} k_{F}^{2}}\right)^{1 / 2}
$$

The first term in Eq. (10) arises from the localization effects, while the second term is the EEI term. Then Eq. (10) can be simply rewritten as,

$$
\sigma(T)=\sigma_{0}+\alpha T^{p / 2}+\beta T^{1 / 2}
$$

We fit Eq. (15) to the experimental conductivity data of the sample. A good agreement is obtained with $p=1.63$ for the sample. Figure 4 shows the conductivity versus temperature accompanying the fitted results (solid line) in the negative $d \rho / d T$ regime. The values of the parameters in Eq. (15) are obtained as $\sigma_{0}=49.6 \pm 0.248$ $(\Omega \cdot \mathrm{cm})^{-1}, \alpha=(1.62 \pm 0.072) \cdot 10^{-2}\left(\Omega^{-1} \cdot \mathrm{cm}^{-1} \cdot \mathrm{K}^{-1}\right)$ and $\beta=0.63 \pm 0.057(\Omega \cdot \mathrm{cm})^{-1} / \mathrm{K}^{1 / 2}$. As expected, the parameter $\alpha$ is quite small in comparison with $\beta$, suggesting that the temperature dependent part of the correction to zero magnetic field conductivity is dominant by the EEI in the investigated sample.

Employing the values of the $D$ and the $\gamma F_{\sigma}$, we can theoretically calculate the value of $\beta$ parameter as 0.65 $(\Omega \cdot \mathrm{cm})^{-1} / \mathrm{K}^{1 / 2}$ for the sample. A satisfactory agreement between the calculated and the fitting (experimental) value of $\beta$ can be clearly seen. This confirms again that the

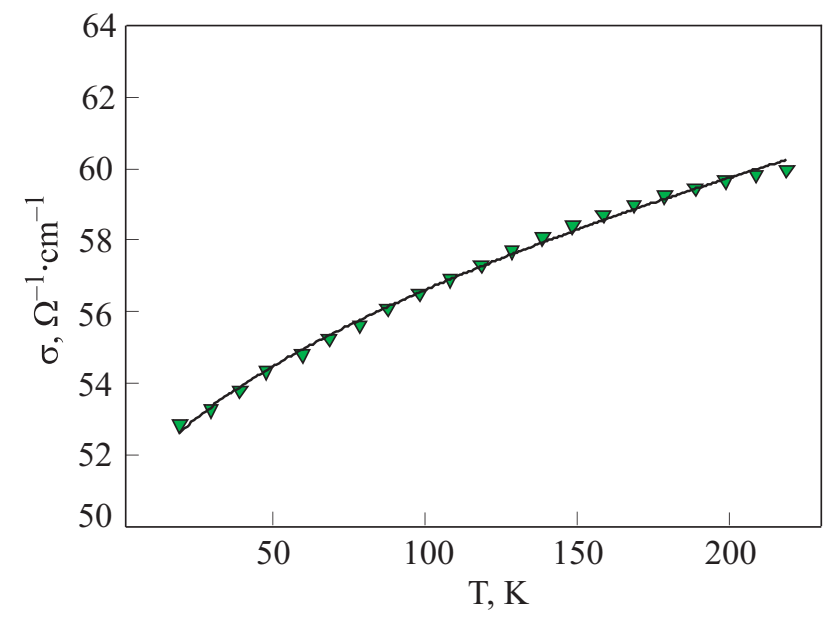

Fig. 4. Temperature dependence of the conductivity for InGaN in the negative $d \rho / d T$ regime. Solid squares show the experimental points, solid line is the best fit with Eq. (15) which includes both the WL and the EEI. inelastic scattering time $\left(\tau_{i}\right)$ has the relationship $\tau_{i} \sim T^{-1.63}$, indicating electron-electron scattering can be considered to be dominant in the sample. Knowing the $D$, the exact temperature dependence of $\tau_{i}$ can be also found as $\tau_{i}=6.59 \cdot 10^{-10} \cdot T^{-1.63} \mathrm{~s}$. The values of $\tau_{i}$ calculated for three temperatures were given in the Table 1. Finally, these values of the $\tau_{i}$ agree with reported values for various materials in the literature [21-25].

\section{Conclusion}

The resistivity, the Hall effect and the MC measurements on an InGaN sample grown by MOVPE have been carried out in a temperature range of $20-350 \mathrm{~K}$. At the low temperatures, the MC was satisfactorily explained by the model, which takes into account WL effect and two-band model. The temperature dependence of inelastic scattering time, $\tau_{i}$, was determined from MC data of the investigated sample. We found that the $\tau_{i}$ has the relationship $\tau_{i} \sim T^{-1.63}$, indicating that the inelastic electron-electron scattering is in dirty limit for the sample. Zero field conductivity data of the sample were well described by the $3 \mathrm{D}$ WL and EEI models in the negative $d \rho / d T$ regime, with parameters that are reasonable agreement with theoretical predictions.

\section{Acknowledgements}

We would like to thank Dr. Mateo Bosi for providing the InGaN sample. This work is supported by the State of Planning Organization of Turkey under Grant No. 2001K120590.

1. S. Nakamura, M. Senoh, N. Iwasa, and S. Nagahama, Jpn. J. Appl. Phys. 34, L797 (1995).

2. M. Asif Khan, A.R. Bhattarai, J.N. Kuznia, and D.T. Olson, Appl. Phys. Lett. 63, 1214 (1993).

3. M. Asif Khan, J.N. Kuznia, D.T. Olson, J.M. Van Hove, M. Blasingame, and L.F. Reitz, Appl. Phys. Lett. 60, 2917 (1993).

4. A. Yildiz, F. Dagdelen, S. Acar, S.B. Lisesivdin, M. Kasap, Y. Aydogdu, and M. Bosi, Acta Phys. Pol. A113, 731 (2008).

5. N.F. Mott, J. Non-Cryst. Solids 1, 1 (1968).

6. A. Yildiz, S.B. Lisesivdin, M. Kasap, and M. Bosi, Solid State Commun. 149, 337 (2009).

7. R. Meseryev and P.M. Tedrow, Phys. Rev. Lett. 41, 805 (1978).

8. E.H. Sondheimer and A.H. Wilson, Proc. R. Soc. London Ser. A 190, 435 (1947).

9. P.A. Lee and T.V. Ramakrishnan, Rev. Mod. Phys. 57, 287 (1985).

10. M. Bosi and R. Fornari, J. Crystal Growth 265, 434 (2004).

11. S.K. Lin, K.T. Wu, C.P. Huang, C.T. Liang, Y.H. Chang, Y.F. Chen, P.H. Chang, N.C. Chen, C.A. Chang, H.C. Peng, C.F. Shih, K.S. Liu, and T.Y. Lin, J. Appl. Phys. 97, 046101 (2005). 
12. S. Aydogu and O. Ozbas, Mat. Sci. Semicon. Proc. 8, 536 (2005).

13. A. Yildiz, S.B. Lisesivdin, S. Acar, M. Kasap, and M. Bosi, Chin. Phys. Lett. 24, 2930 (2007).

14. A. Kawabata, J. Phys. Soc. Jpn. 50, 2131 (1981).

15. D.V. Baxter, R. Richter, M.L. Trudeau, R.W. Cochrane, and J.O. Strom-Olsen, J. Phys. (Paris) 50, 1673 (1989).

16. A. Schmid, Z. Phys. 271, 251 (1974).

17. N.F. Mott and M. Kaveh, Adv. Phys. 34, 329 (1985).

18. N.F. Mott and M. Kaveh, Philos. Mag. B52, 177 (1985).

19. B.L. Altshuler and A.G. Aronov, Solid State Commun. 46, 429 (1983).
20. B.L. Altshuler and A.G. Aronov, in: Electron-Electron Interactions in Disordered Systems, A.L. Efros and M. Pollak (eds.), North-Holland, New York (1985).

21. A.F. Brana, C. Diaz-Paniagua, F. Battalan, J.A. Garrido, E. Munoz, and F. Omnes, J. Appl. Phys. 88, 932 (2000).

22. N. Thillosen, Th. Schäpers, N. Kaluz, H. Hardtdegen, and V.A. Guzenko, Appl. Phys. Lett. 88, 022111 (2006).

23. Z.W. Jia, W.Z. Shen, H. Ogawa, and Q.X. Guo, Appl. Phys. Lett. 89, 232107 (2006).

24. A.S. Troup, J. Wunderlich, and D.A. Williams, J. Appl. Phys. 101, 033701 (2007).

25. T. Kawazu and H. Sakaki, Physica E29, 593 (2005). 